\title{
Avoidable hospitalization of children from ambulatory care sensitive conditions in Barbados: a measure of the access and uptake of primary health care
}

\author{
Alok Kumar ${ }^{1 *}$, Gemma Ann Benskin ${ }^{2}$ and Richa Sharma² \\ ${ }^{1}$ The Faculty of Medical Sciences, The University of the West Indies (Cave Hill), Barbados \\ ${ }^{2}$ The Queen Elizabeth Hospital, Barbados
}

\begin{abstract}
Background and Objective: Hospitalization from avoidable causes refers to hospitalizations for an Ambulatory Care Sensitive (ACS) condition and is considered to be a measure of access to appropriate primary health care. While not all admissions for these conditions are avoidable, timely ambulatory care could temper the disease course and thus prevent progression to where hospitalization is medically indicated. A disproportionately high rate of ACS conditions may reflect problems in access to timely primary care. In this study we determine the proportion of all medical admissions in children that were from ACS Conditions (ASSC's) and therefore avoidable. We also study the trend in the proportion of avoidable hospitalizations.

Method: This study is a population based prospective clinical audit of children (Age <16 years) admitted to the Queen Elizabeth Hospital (QEH). The study period extends from the year 2013 through 2017. The pediatric-specific ACS conditions employed in this analysis were based on prior studies. All the children between the ages of 3 month and 15 years where one of the discharge diagnosis was an ACS condition were included in this study.

Result: During the five years study period there were a total of 19349 children ( $<16$ years) hospitalized for medical conditions to the QEH. Over the same period, there were a total of 4235 hospitalizations from ACSC's which accounted for $21.89 \%$ of all medical hospitalizations in children. Among the ACSC's as the cause of hospitalization, acute asthma (6.88\%) and acute asthma complicated by respiratory tract infections (2.24\%) was the commonest cause for avoidable hospitalizations in children. This was followed by admissions from acute gastroenteritis and dehydration (2.89\%). Children less than 4 years in age accounted for $51 \%$ of all avoidable medical hospitalizations in children.
\end{abstract}

Conclusion: Over a fifth of all the medical hospitalizations in children are from the ACSC's and are therefore avoidable.

\section{Introduction}

Hospitalization from avoidable causes refers to hospitalizations for an Ambulatory Care Sensitive (ACS) condition and is considered to be a measure of access to appropriate primary health care [1]. Ambulatory care-sensitive Conditions (ACSC's) are conditions for which high quality outpatient care can potentially prevent the need for hospitalization [1]. ACSCs were developed by expert consensus methods, have substantial face validity, [2,3] and account for up to onethird of pediatric hospitalizations [4]. Examples of pediatric ACSCs include asthma, urinary tract infection, and dehydration.

While not all admissions for these conditions are avoidable, timely ambulatory care could temper the disease course and thus prevent progression to where hospitalization is medically indicated. A disproportionately high rate of ACS conditions may reflect problems in access to timely primary care [5]. ACS conditions have been previously described for use as a measure of potentially preventable hospitalizations $[1,4,6,7]$. Studies using these indicators have enabled the improvement of actions and public policies capable of reducing the childhood morbidity and improving the health and wellbeing of children and family $[5,8]$. The high frequency of hospitalizations due to diseases considered to be easily preventable, which can be diagnosed and treated, reflect either a less than adequate primary health care delivery or utilization by the population, as well as their poor life and health conditions or both [5].
With increasing concerns about healthcare spending gains prominence, healthcare providers and legislators need to examine areas where healthcare expenditures can be reduced without compromising the quality of care. One of the solutions to limit healthcare spending involves reducing the number of preventable hospitalizations [8].

Barbados one of the English-speaking Caribbean countries has a pluralistic health care system. It has a comprehensive public health care system which provides all level of care free of charges at the point delivery. There is a parallel private health care delivery facilities where payment is from personal or group health insurance or out of pocket. Studies on causes of hospitalization among children in Barbados is facilitated by the good record keeping at its only tertiary health care hospital which provides for all of the hospitalization in this country. In this study we determine the proportion of all medical admissions in children that were from ACSC'S and therefore avoidable. We also study the trend in the proportion of avoidable hospitalizations.

${ }^{\star}$ Correspondence to: Professor Alok Kumar, Faculty of Medical Sciences at The Queen Elizabeth Hospital, Martindales Road, Bridgetown St. Michael, Barbados, E-mail: alok.kumar@cavehill.uwi.edu; alokkumar.uwichill@gmail.com

Received: October 12, 2020; Accepted: October 19, 2020; Published: October 22, 2020 


\section{Methods}

This study is a population based prospective clinical audit of children (Age $<16$ years) admitted to the Queen Elizabeth Hospital (QEH). The study period extends from the year 2013 through 2017. A preplanned analysis of data from review of medical records was used to characterize all children requiring admission for ACS conditions. The QEH is the only tertiary care hospital in Barbados where children are admitted in this country. Each patient were treated at the discretion of the treating physician. The international Classification of Diseases IX revision was be used to revise the discharge diagnosis based on the clinical presentations and the results from the laboratory investigations, where necessary, during the review of the individual clinical records.

Potentially preventable pediatrics hospitalizations were defined through the use of ambulatory care-sensitive (ACS) conditions. The pediatric-specific ACS conditions employed in this analysis were based on a prior studies [6,7].

The pediatric medical hospitalization register at the QEH were used to identify all admissions in children where an ACS condition was the primary discharge diagnosis. All the children between the ages of 3 month and 15 years where one of the discharge diagnosis was an ACS condition were included in this study. The threshold of 3 months removed neonatal admissions as well as young infants who typically have a lower threshold for hospital admission. Pooled data such as overall number of admissions to the pediatric medical ward and number of admissions for ARD for each months during the study period were also collected. Data on the population demographics in Barbados were also be collected for the study time period.

Outcome variables of interest included number of ACS admissions, determined by whether the primary discharge diagnosis was one of the ACS codes; total number of hospitalizations in children. Demographic factors examined included gender and age.
Descriptive analyses examined demographic characteristics of ACS hospitalizations. Bivariate analysis, including chi-square for categorical variables, t-test for continuous variables associated with an ACS hospitalization, that were statistically significant at the $\mathrm{p}<0.05$ level.

\section{Results}

During the five years study period there were a total of 19349 children $(<16$ years) hospitalized for medical conditions to the QEH. Figure 1 shows the overall medical hospitalizations among children in Barbados. The mean annual hospitalization among children during the study period was 3870 (Range 3744 - 4033). Over the same period, there were a total of 4235 hospitalizations from ACSC's which accounted for $21.89 \%$ of all medical hospitalizations in children. Among the children who hospitalized with the ACSC's, $45 \%$ were females and $55 \%$ were males. The age distribution of children hospitalized for one of ACSC's is shown in Figure 2. Children less than 4 years in age accounted for $51 \%$ of all medical hospitalizations in children. Trends in the proportion of hospitalizations from ACSC's is shown in Figure 1 and 2. During the five year study period, the proportion of all hospitalizations that resulted from ACSC's decreased from 23\% in 2013 to $20 \%$ in 2017. However, the difference in the proportion of hospitalizations that were from ACSC's during the different years were statistically not significant.

The hospitalizations among children's from different ACSC's are shown in Table 1. Among the ACSC's as the cause of hospitalization, acute asthma $(6.88 \%)$ and acute asthma complicated by respiratory tract infections (2.24\%) were the commonest cause for hospitalizations. Acute respiratory diseases including asthma and respiratory tract infections accounted for $11.85 \%$ of all medical hospitalizations in children. This was followed by admissions form acute gastroenteritis and dehydration (2.89\%). Hospitalizations form different acute respiratory diseases and its trends over the study period is shown in Figure 3. There were no significant trends seen in the hospitalizations from any of the acute respiratory diseases (Figure 4).

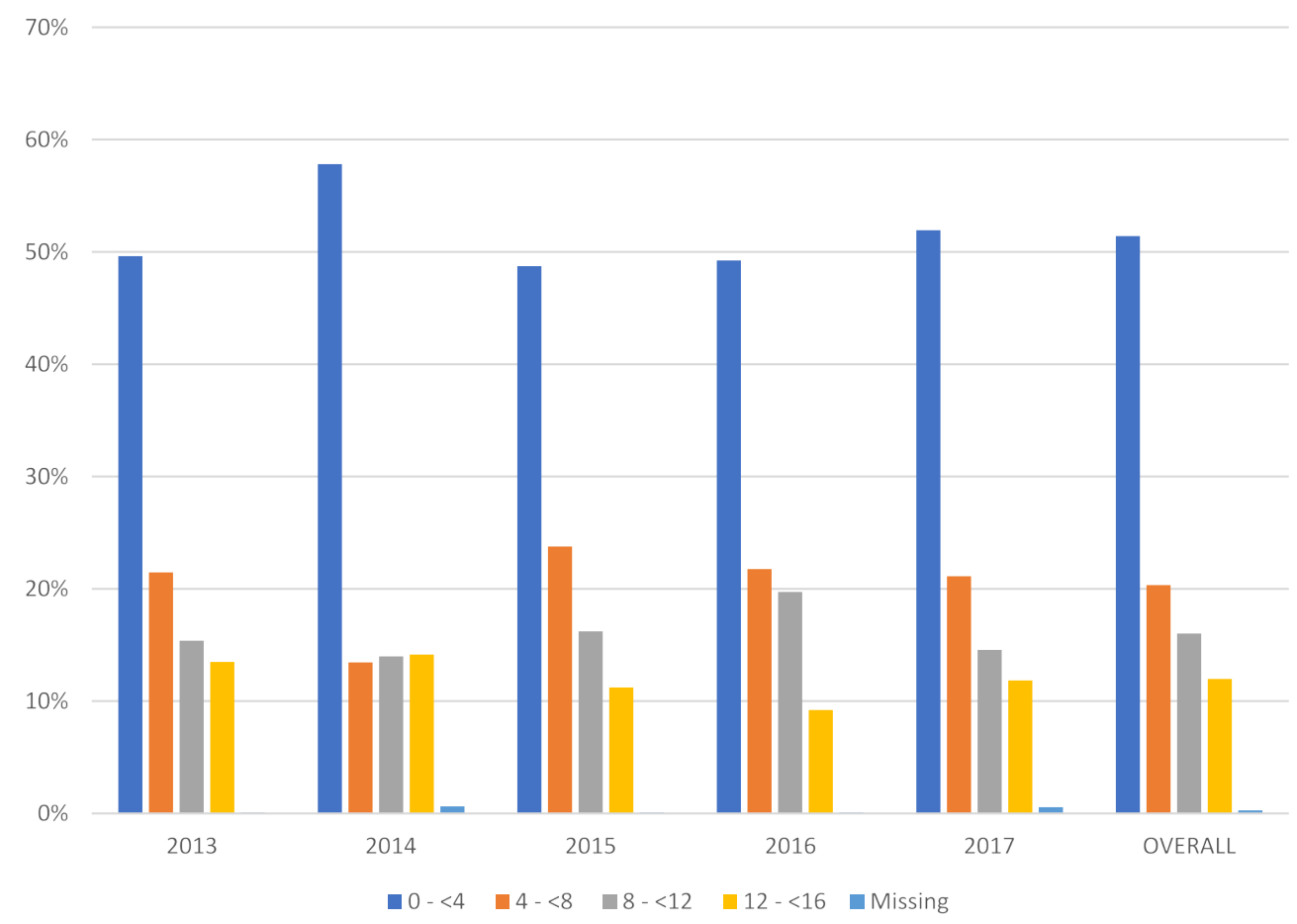

Figure 1. Age distribution of children hospitalized for medical conditions in Barbados, 2013-2016 


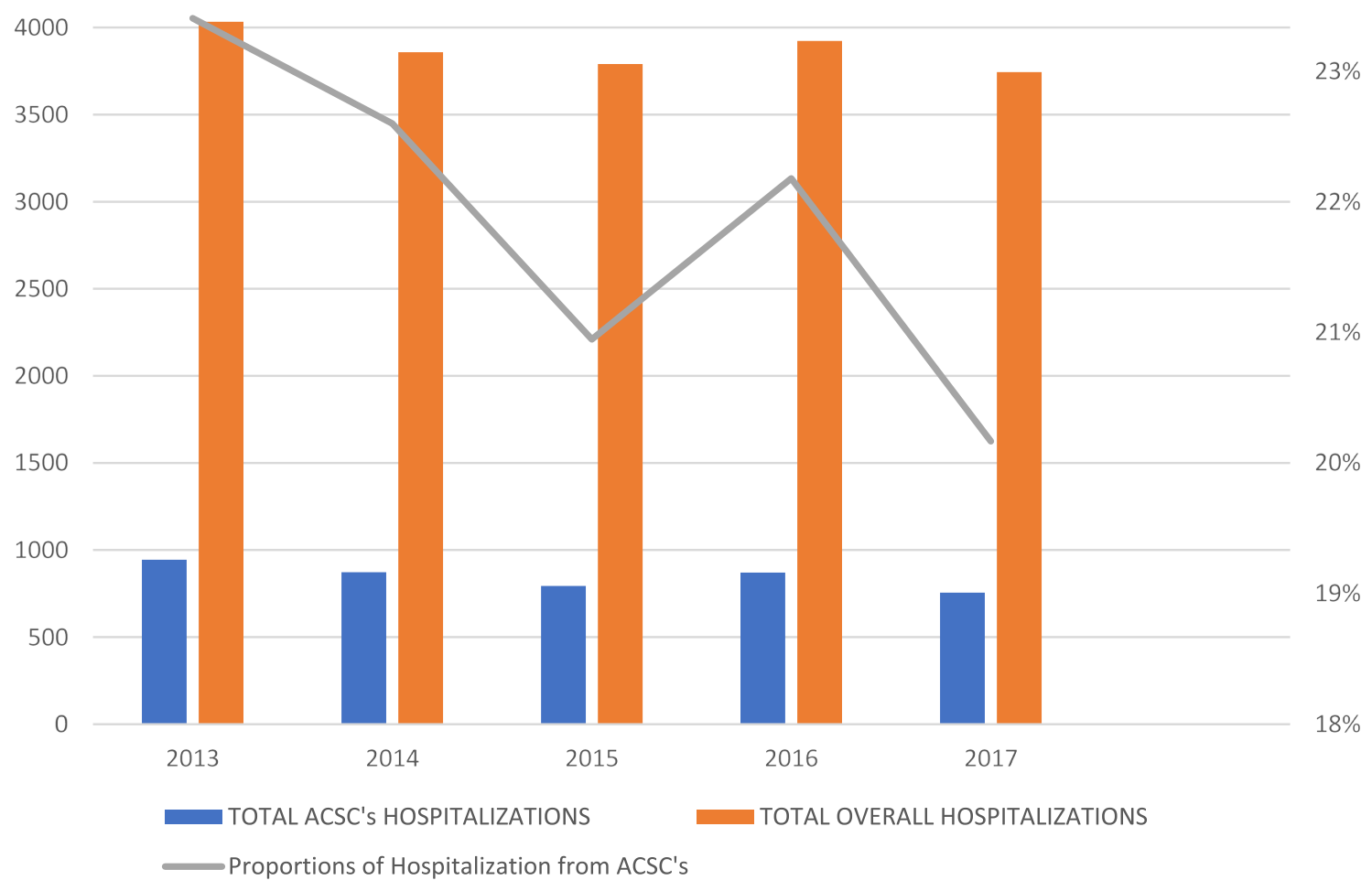

Figure 2. Trends in the proportions of hospitalizations among children from ACSC's causes in Barbados, 2013-2017

700

600

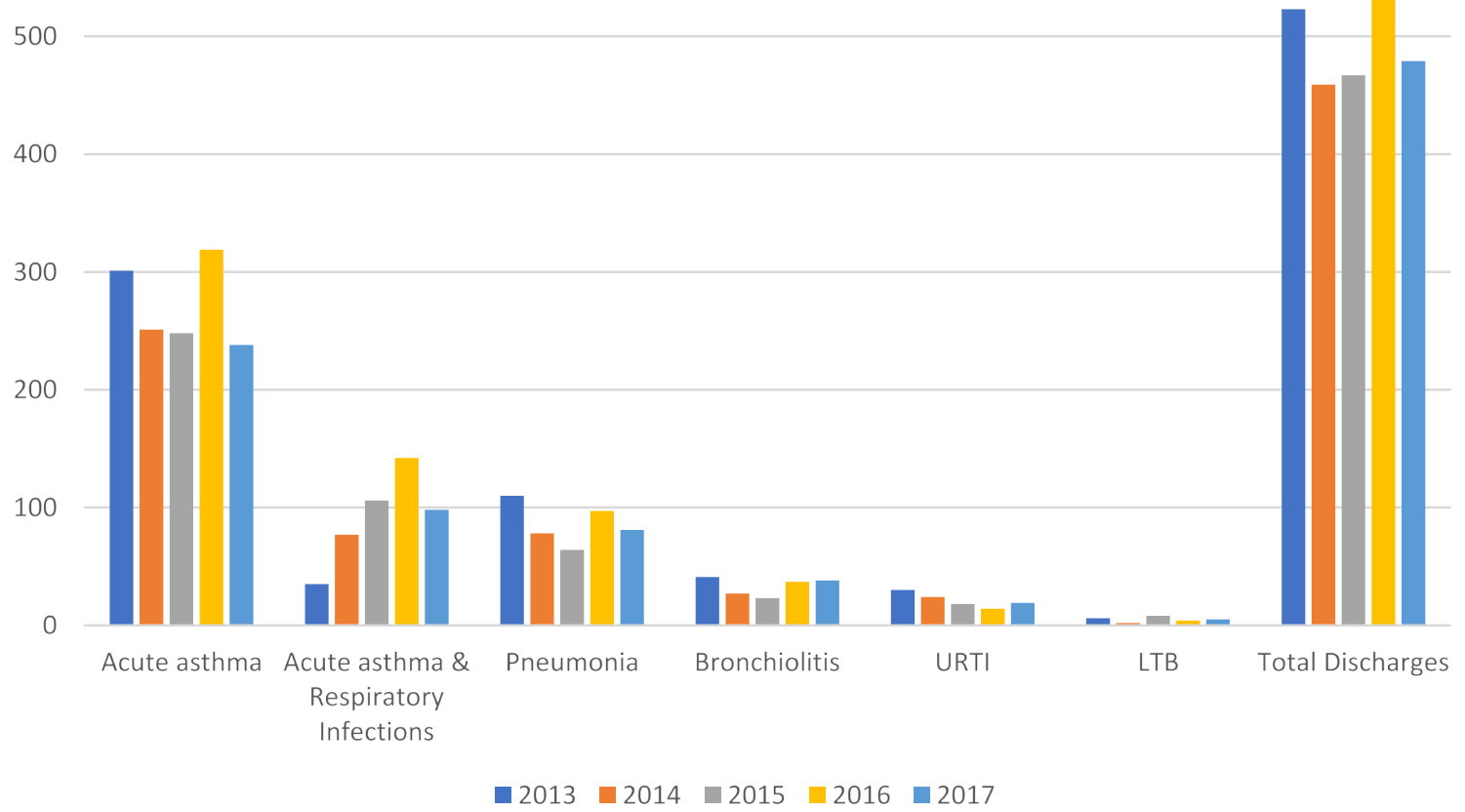

Figure 3. Trends in the hospitalization from various acute respiratory diseases among children in Barbados, 2013-2017 


\section{Trends in admissions from acute respiratory diseases among children in Barbados, 2013-2017.}

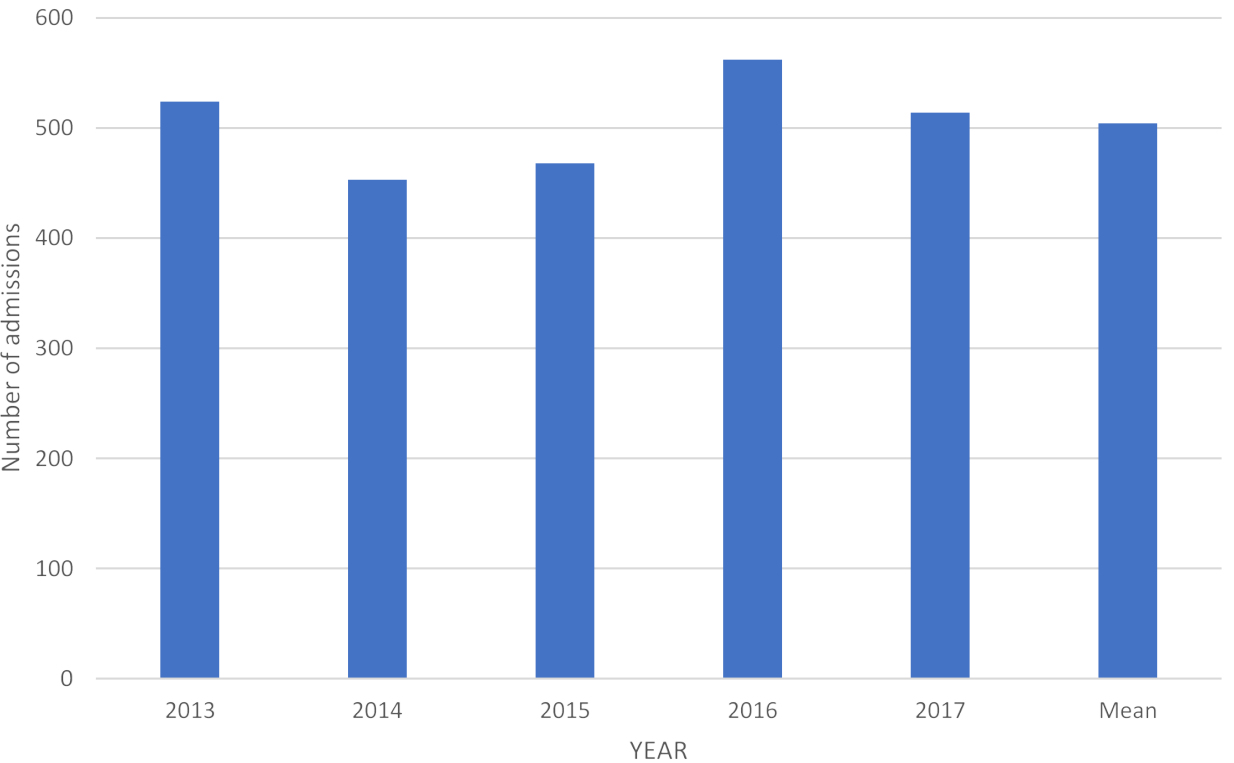

Figure 4. Trends in admissions from acute respiratory diseases among children in Barbados, 2013-2017

Table 1. Major ACSC's causes for hospitalizations among children in Barbados, 2013-2017

\begin{tabular}{|c|c|c|c|c|c|c|c|}
\hline ACSC's Diagnosis & 2013 & 2014 & 2015 & 2016 & 2017 & $\begin{array}{c}\text { Total } \\
\text { Hospitalization }\end{array}$ & $\begin{array}{c}\text { Proportion of } \\
\text { mean annual } \\
\text { admissions }\end{array}$ \\
\hline Urinary tract Infection & 10 & 13 & 17 & 27 & 29 & 96 & $0.50 \%$ \\
\hline Diabetes mellitus & 14 & 15 & 12 & 21 & 22 & 84 & $0.43 \%$ \\
\hline Upper respiratory tract infection & 30 & 24 & 17 & 14 & 19 & 104 & $0.54 \%$ \\
\hline Acute Asthma \& Respiratory infection & 35 & 77 & 106 & 117 & 98 & 433 & $2.24 \%$ \\
\hline Sickle cell disease & 56 & 42 & 54 & 47 & 43 & 242 & $1.25 \%$ \\
\hline Pneumonia & 110 & 78 & 64 & 90 & 81 & 423 & $2.19 \%$ \\
\hline Acute viral illness & 113 & 167 & 95 & 80 & 66 & 521 & $2.69 \%$ \\
\hline Seizure disorder & 122 & 111 & 63 & 76 & 69 & 441 & $2.28 \%$ \\
\hline Acute gastroenteritis & 153 & 94 & 118 & 104 & 90 & 559 & $2.89 \%$ \\
\hline Acute Asthma & 301 & 251 & 248 & 294 & 238 & 1332 & $6.88 \%$ \\
\hline TOTAL ACSC's HOSPITALIZATIONS & 944 & 872 & 794 & 870 & 755 & 4235 & $21.89 \%$ \\
\hline TOTAL OVERALL HOSPITALIZATIONS & 4033 & 3859 & 3791 & 3923 & 3744 & 19349 & $100.00 \%$ \\
\hline
\end{tabular}

\section{Discussion}

Ambulatory Care Sensitive Conditions (ACSC), also known as Potentially Avoidable Paediatric Hospitalizations (PAPH) when related to children and adolescents, are conditions for which hospitalization is thought to be avoidable with the application of effective preventive care and early disease management, which is usually delivered in a primary care setting. Avoidable hospitalization rates have been used as quality indicators of healthcare provided to populations, as access indicators of primary care, and as indicators of outpatient care related to primary care capacity. These indicators, based on the fact that appropriate use of ambulatory care services can reduce hospitalization, can help to expand policies and programs related to morbidity and mortality, which can decrease the costs of second and third level health care and improve patient quality of life by decreasing patient suffering $[9,10]$.

The QEH is the only tertiary care hospital in Barbados which provides facility for the hospitalizations for children. Therefore, the findings of this study on the preventable hospitalizations from the conditions defined as the ACSC's is reflective of the whole population. This is the first such long term study form Barbados and the wider Caribbean and therefore comparisons of data was impossible. Barbados had an estimated under 16 population of 51165 in 2015 [11]. With a mean annual hospitalization of 3870 , Barbados has a higher rate $(7.56 \%)$ of hospitalization in under 16 children excluding hospitalization in newborn babies at delivery compared to those from North America [12].

Over one-fifth of all medical hospitalization in children $(<16$ years) resulted from ACSC's and were therefore avoidable. This proportion is higher than those reported (14.1\%) form the USA [13]. Respiratory disease accounted for much of the avoidable admissions as also reported in several other studies $[7,9,13]$. Hospitalization form Asthma was one of the most common avoidable condition and needs urgent attention. 
In conclusion, over one fifth of all hospitalization among children in this country is avoidable. Asthma accounts for the most common avoidable hospitalization in children.

\section{Reference}

1. Billings J, Zeitel L, Lukomnik J, Carey TS, Blank AE, et al (1993) Impact of socioeconomic status on hospital use in New York City. Health Aff (Millwood) 12: 162173. [Crossref]

2. McDonald K, Romano P, Davies S, Haberland C, Geppert J, et al. (2006) Measures of Pediatric Health Care Quality Based on Hospital Administrative Data. The Pediatric Quality Indicators pp: 1-137.

3. Sanderson C, Dixon J (2000) Conditions for which onset or hospital admission is potentially preventable by timely and effective ambulatory care. J Health Serv Res Policy 5: 222-230. [Crossref]

4. Lu S, Kuo DZ (2012) Hospital charges of potentially preventable pediatric hospitalizations. Acad Pediatr 12: 436-444. [Crossref]

5. National Quality Measures Clearinghouse (2019) Ambulatory care sensitive conditions. Age-standardized acute care hospitalization rate for conditions where appropriate ambulatory care prevents or reduces the need for admission to the hospital, per 100,000 population under age 75. Ambulatory Care Sensitive Conditions.
6. Garg A, Probst JC, Sease T, Samuels ME (2003) Potentially preventable care: ambulatory care-sensitive pediatric hospitalizations in South Carolina in 1998. South Med J 96: 850-858. [Crossref]

7. Shi L, Samuels ME, Pease M, Bailey WP, Corley EH (1999) Patient characteristics associated with hospitalizations for ambulatory care sensitive conditions in South Carolina. South Med J 92: 989-998. [Crossref]

8. Institute of Medicine (US) (2009) State of the USA Health Indicators. State of the USA Health Indicators: Letter Report. National Academies Press (US), Washington (DC).

9. Casanova C, Colomer C, Starfield B (1996) Pediatric hospitalization due to ambulatory care-sensitive conditions in Valencia (Spain). Int J Qual Health Care 8: 51-59. [Crossref]

10. Ansari Z, Haider SI, Ansari H, Gooyer T, Sindall C (2012) Patient characteristics associated with hospitalisations for ambulatory care sensitive conditions in Victoria, Australia. BMC Health Serv Res 12: 475. [Crossref]

11. https://www.worldometers.info/demographics/barbados-demographics/\#median-age

12. Witt WP, Weiss AJ, Elixhauser A (2014) Overview of Hospital Stays for Children in the United States, 2012. HCUP Statistical Brief \#187. Agency for Healthcare Research and Quality, Rockville, MD.

13. Medford-Davis LN, Shah R, Kennedy D, Becker E (2016) Factors Associated With Potentially Preventable Pediatric Admissions Vary by Diagnosis: Findings From a Large State. Hosp Pediatr 6: 595-606. [Crossref]

Copyright: $\odot 2020$ Kumar A. This is an open-access article distributed under the terms of the Creative Commons Attribution License, which permits unrestricted use, distribution, and reproduction in any medium, provided the original author and source are credited. 